\title{
SPHERICALLY SYMMETRIC KINEMATIC MODELLING OF PLANETARY NEBULAE
}

\author{
C. DIESCH (1) and M. GREWING $(1,2)$ \\ (1)Astronomisches Institut der Universität \\ Waldhäuserstraße 64, D-7400 Tübingen \\ (2)Institute for Radioastronomy at Millimeter Wavelengths (IRAM) \\ 300, rue de la Piscine,Domaine Universitaire,F-38406 St.Martin d'Hères, France
}

\begin{abstract}
High resolution two-dimensional long slit spectra of NGC 7009, NGC 3242 , IC 2448 and TC 1 in the $\mathrm{H} \alpha(6565 \AA), \operatorname{OIII}(5007 \AA)$ and NII(6584 $\AA)$ emission lines have been used as input to determine radial velocity gradients in $\mathrm{PNe}$ by fitting calculated high resolution spatially resolved spectral structures of single emission lines. Contributions to the Doppler structure of emission lines taken into account are the global expansion of the PN shell and thermal line broadening due to temperatures of some $10^{4} \mathrm{~K}$. This model is based on two parametrized empirically chosen spherical symmetric functions:
\end{abstract}

1. A empirical two parameter density function $\rho(r)$.

2. A linear radial velocity component function $v(r)$.

Fitting this model to the observed PN spectra yield the following results: All observed PNe show increasing radial velocity towards the outer rim of the shell. The velocity function $v(r)$ derived for NGC 7009 fits excellent to the former results for the outer regions of this PN (Weedman). In the case of TC 1 the velocity $v(r)$ function derived for different radii from OIII, $\mathrm{H} \alpha$, and NII due to the ionisation structure in the PN are in very good agreement. The table below lists these results numerically:

\begin{tabular}{|l|l|r|r|r|}
\hline & line & $\begin{array}{r}\mathrm{v}(\mathrm{r}=0) \\
{[\mathrm{km} / \mathrm{s}]}\end{array}$ & $\begin{array}{r}\mathrm{dv} / \mathrm{dr} \\
{\left[\mathrm{km} / \mathrm{s} / 10^{12} \mathrm{~km}\right]}\end{array}$ & $\begin{array}{r}\text { appl. radii } \\
{\left[10^{12} \mathrm{~km}\right]}\end{array}$ \\
\hline NGC 7009 & $\mathrm{H} \alpha$ & -4.1 & 7.3 & $1.5-4.5$ \\
NGC 3242 & $\mathrm{H} \alpha$ & -28.2 & 11.4 & $2.5-4.5$ \\
IC 2448 & $\mathrm{H} \alpha$ & 4.5 & 3.6 & $0-5.0$ \\
TC 1 & $\mathrm{OIII}$ & -1.2 & 3.7 & $0-2.5$ \\
& $\mathrm{H} \alpha$ & -7.8 & 6.4 & $1.0-4.5$ \\
& NII & -9.1 & 6.5 & $1.5-5.5$ \\
\hline
\end{tabular}

\section{References}

Weedman, D., Astrophys. J. 153, 49

Weinberger, R., Astron. Astrophys. Suppl. Ser. 78, 301 